\title{
ELECTROPHYSIOLOGICAL PROPERTIES OF THE CANINE VENTRICULAR FIBER
}

\author{
Akimitsu Kamiyama And Kōjiro Matsuda \\ Department of Physiology, Faculty of Medicine, University \\ of Tokyo, Tokyo
}

In the mamalian ventricle there are two kinds of muscle fibers: the Purkinje fiber and the proper ventricular fibers. While the electrophysiological properties of the Purkinje fiber have been systematically studied by several investigators $^{6,9,12,15,16)}$, as to the ventricular fiber most researches appeared to be incomplete or fragmentary $y^{1,2,3,6,7,8,9,11,14)}$, probably because its complicated architectures with extensive branching and anastomosing hampered the recording of reasonable electrotonic potentials essential to analysis. And almost all of these investigations seem to have demonstrated that the electrophysiological characteristics of the ventricular fiber are, by and large, different from those of the Purkinje fiber.

It was the authors' experience that in the ventricular muscle, contrary to expectation, the routine procedure for polarizing the single fiber by means of an intracellular microelectrode could not produce electrotonic potentials of reasonable size and spatial spread in the adjoining fibers. It may be due to the fact that, as MATSUDA pointed out ${ }^{10}$, the mode of excitation and conduction in the cardiac muscle and the change of its membrane potential, active as well as passive, can be affected more or less by the presence of adjacent cells and their functional states.

The present communication is concerned with the results of experiments in which a newly designed arrangement enabled the authors to record the electrotonic potentials in the ventricular fibers, and to obtain some of the informations about the electrophysiological properties of its membrane.

\section{METHOD}

Sample: All experiments were carried out in the papillary muscle of the dog. In the free-end portion of it, all the subendocardial cells are the typical ventricular fibers from the first layer, as being judged by the form of action potential. Histological examination by a special technique which identified the cell impaled by the micro-

Received for publication December 25, 1965

上山章光, 松田幸次郎 


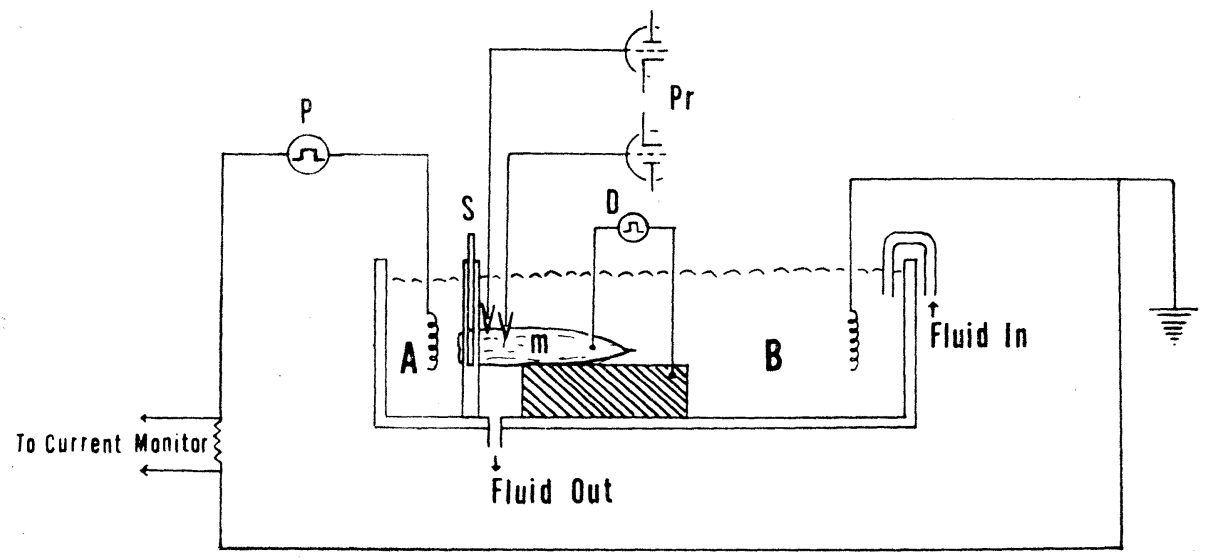

FIG. 1. Diagram of experimental arrangement. Papillary muscle (m) taken from the right ventricle of canine heart is held compact at the partition (S) of two chambers $\mathrm{A}$ and $\mathrm{B}$. Bathing fluids are isotonic $\mathrm{KCl}$ in $\mathrm{A}$, oxygenated Tyrode solution in $\mathrm{B}$. D : Driving pulse generator. Stimulator (P) for polarizing current is coupled with (D) via delay circuit. Pr: Preamplifier with high input impedance.

electrode (the method to be published elsewhere) also ascertained it.

Experimental arrangement: The sample was fixed in a specially designed separation box made of Lucite. The box was divided in two chambers $A$ and $B$ as is shown in FIG. 1. The sample, set on the boundary of the two chambers, was fixed compactly between a thin celluloid plate ( $\mathrm{S}$ in FIG. 1, $0.3 \mathrm{~mm}$ in thickness) and the partition of the chambers. The radicle of the papillary muscle which was placed in chamber A was chipped as close as possible to the partition with a razor blade. Chamber $B$ was perfused with oxygenated Tyrode's solution of $37^{\circ} \mathrm{C}$, while the chamber $\mathrm{A}$ was filled with isotonic KCL solution. The sample was driven electrically at a constant rate (interval: $800 \mathrm{msec}$ ) through a bipolar $\mathrm{Ag}-\mathrm{AgCl}$ electrode which was placed on the sample in chamber $B$. The polarizing current was applied between chamber $A$ and $B$ through $\mathrm{Ag}-\mathrm{AgCl}$ electrodes put in both chambers respectively, with minimized leakage around the sample. The current produced electrotonic potentials in the muscle which were recorded at various distances from the partition. In such arrangement, bundles of numerous muscle fibers were found to undergo a uniform polarization as if in the polarization of an isolated single fiber, presumably the effects of frequent branching and re-uniting (anastomosing) of fibers in the course of longitudinal direction cancelled each other. Pulses for polarization were supplied from an electronic stimulator (Type (MSE-3, Nihon-Koden Co.,) through an isolator with a definite time delay following the driving stimulus.

In recording the membrane potential, to minimize the artifact of the polarizing current, the inputs from two similar microelectrodes, one inside and the other immediatedly outside the fiber membrane to the examined, were fed into a differential preamplifier. By careful adjustment of the position of the extracellular electrode, the artifact could effectively be cancelled out. Resistances of the microelectrodes used ranged between 5 and 10 megohms. Both the membrane potential and the polarizing current were always monitored simultaneously on the same screen of a dual-beam cathode ray oscilloscope. 


\section{RESULTS}

For a while after setting the preparation and in the area close to the partition (within 1 to $2 \mathrm{~mm}$ ), the resting potential was low and the shape of induced action potential was abnormal, propably owing to an injury given to the cut end. But in most cases such effect subsided gradually within 30 minutes. As a rule, the experimental observation was started about one hour after setting the preparation when the normal resting as well as action potentals had been resumed.

Electrotonic potentials When rectangular anodal current of $50 \mathrm{msec}$ duration was passed from chamber $B$ to chamber $A$ in the diastolic phase between repetitively induced action potentials, distinct electrotonic potentials could be observed in various sites along the longitudinal axis of the sample, from the partition toward the free end of muscle. The amplitude of this electrotonic potential which was largest at the most proximity to the partition, decayed with distance. The shapes and amplitudes of such electrotonic potentials are shown in FIG. 2. The decay of amplitude with distance was justly exponential as is shown in FIG. 3. The rate of this decay was a little greater than that in the Purkinje fiber. Consequently the space constant $(\lambda)$ which was estimated to be $1.3 \mathrm{~mm}$ from the decay curves (average of 5 experiments) was slightly less than that for the Purkinje fiber $(1.9 \mathrm{~mm})^{16)}$. (TABLE 1 ).

The half time of rising phase of electrotonic potentials is in a nearly linear relationship to the distance from the partition (FIG. 4). According to the observations on nerve fibers by HoDGKIN and RUSHTON ${ }^{5)}$, the half time increased linearly with distance from the site of current source, and the slope of this linearity was nearly equal to $2 \lambda / \tau$, here $\tau$ denoting the time constant of the membrane. Also in the present observation, such linear relation was clearly noticed; and the time constant $(\tau)$ of the membrane of the ventricular fiber, calculated from the slope, was $2.0 \mathrm{msec}$ (average of 5 experiments) (TABLE 2).

TABLE 1. Space constant " $\lambda$ " and time constant " $\tau$ " of the membrane in ventricular fiber of dog.

\begin{tabular}{c|c|c|c|c|c|c}
\hline No. of experiment & 1 & 2 & 3 & 4 & 5 & mean \\
\hline Space constant “ $\lambda$ " $(\mathrm{mm})$ & 1.01 & 1.43 & 1.46 & 1.50 & 1.34 & 1.35 \\
\hline Time constant " $\tau$ " $(\mathrm{msec})$ & 1.7 & 1.7 & 2.3 & 2.3 & 2.0 & 2.0 \\
\hline
\end{tabular}

TABLE 2. Threshold potential of ventricular fiber of dog.

\begin{tabular}{c|c|c|c|c|c|c}
\hline No. of experiment & 1 & 2 & 3 & 4 & 5 & mean \\
\hline Threshold potential $(-\mathrm{mV})$ & 65 & 65 & 60 & 62 & 75 & 65.4 \\
\hline
\end{tabular}


A. KAMIYAMA AND K. MATSUDA
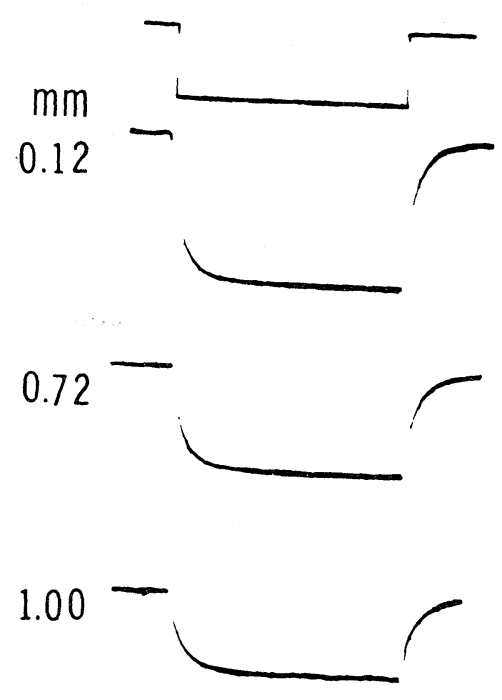

FIG. 2. Change in electrotonic potential at various distances from the partition. Uppermost curve shows polarizing current.
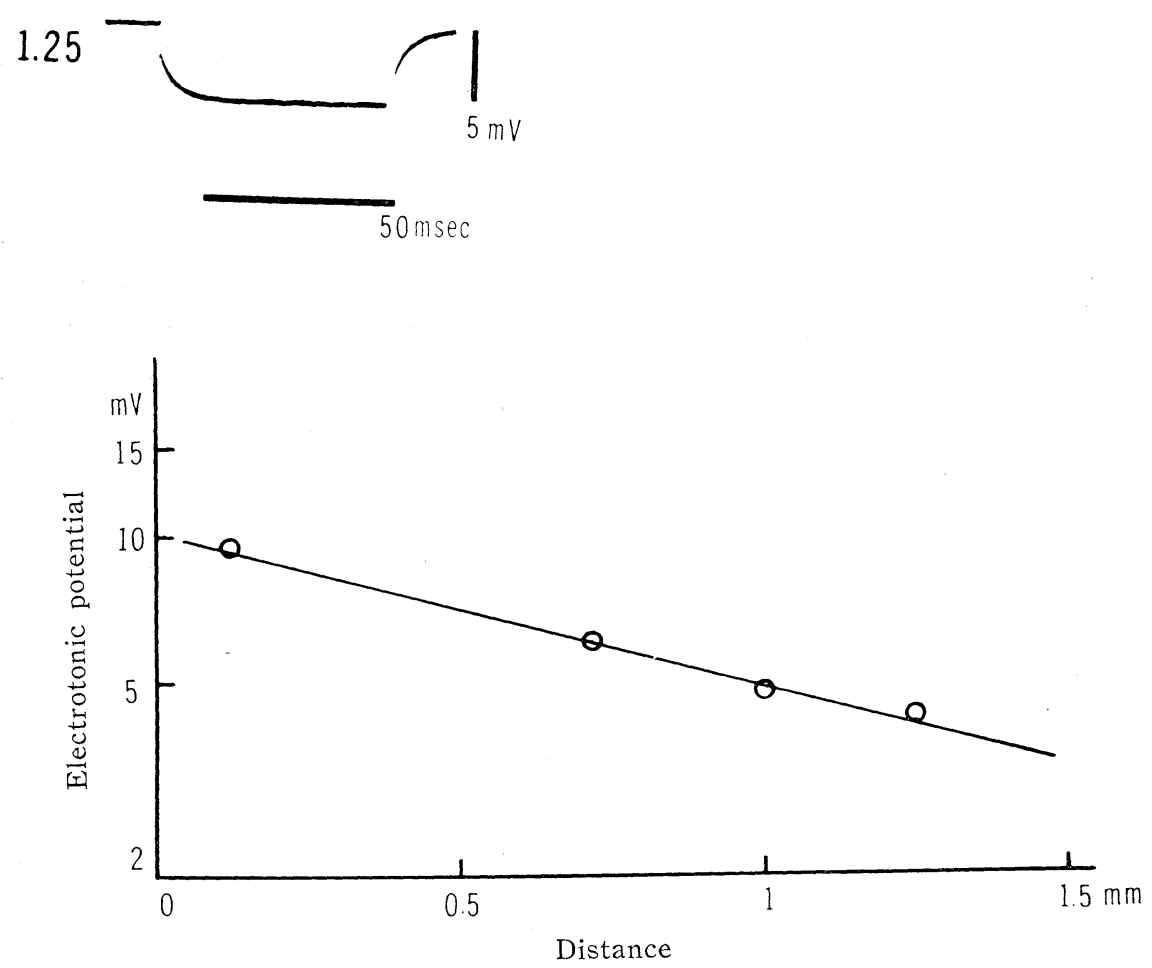

FIG. 3. Decay of electrotonic potential with distance. Abscissa: Distance from partition. Ordinate : Amplitude of electrotonic potential, in logarithmic scale. 


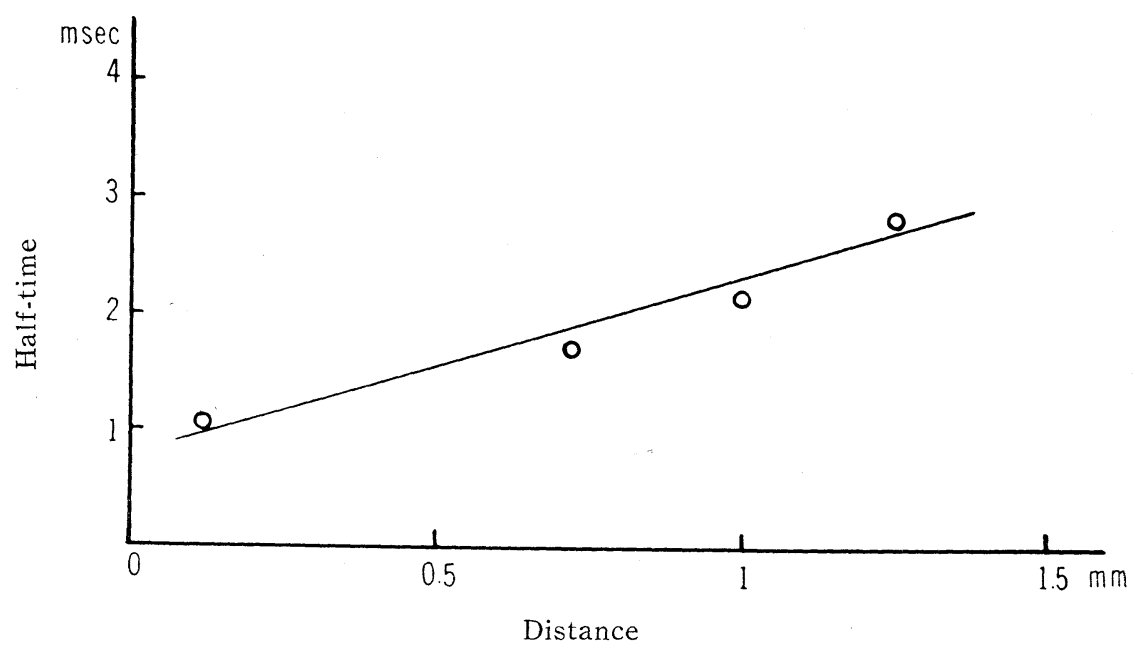

FIG. 4. Approximately linear relation of half-time of electrotonic potential to distance. Abscissa: Distance from partition. Ordinate: Half-time of electrotonic potential.

Critical membrane potential By the same procedure as above, the intensity of cathodal current was increased so that the resultant depolarization attained the firing level of the fiber. The critical membrane potential can not be estimated straightforwardly from the potential where the rising phase of action potential appeared to be touched off, for this level of potential varied depending on the distance from the partition to the recording site. At sites far from the partition, the transition from the passive electrotonic potential to the regenerative depolarization was not gradual but abrupt, and the potential at its sudden rise was higher (in negativity) than the true critical potential. The latter could be estimated in the records taken from the cells being situated as close as possible to the partition and yet having the normal resting and action potentials (FIG. 5). The value of the critical membrane potential thus estimated was $-65 \mathrm{mV}$ (average of 5 experiments) (TABLE 2).

Changes in membrane resistance in action potential Change in membrane resistance at the crest of action potential could be judged by comparing amplitudes of electrotonic potentials produced by a weak anodal current applied at that phase and in diastole. In the Purkinje fiber, it was already ascertained that the value of membrane potential just before the onset of the upstroke of action potential affects the height of spike ${ }^{17}$. In view of this fact, in the present study on the ventricular fiber, the heights of spike peaks were compared in two cases, namely: in the one case where the application just before the upstroke of action potential, and in the other where the current of the same intensity and duration was passed a little later so that it covered the spike and initial portion of plateau. In FIG. 6-A the electrotonic potential was 
$10 \mathrm{mV}$ during the resting phase, and the overshoot of action potential was $29 \mathrm{mV}$. In FIG. 6-B, where the same pulse was shifted a little later as mentioned above, the overshoot was $20 \mathrm{mV}$. FIG. 6-C where both figures $\mathrm{A}$ and $B$ are represented superimposed, shows that the electrotonic potential at the crest of action potential was only slightly less $(-10 \%)$ than that at the resting phase. In many other cases, the differences were still less, or hardly detectable. Therefore, the decrease in membrane resistance at the spike peak seems to be quite small, if any, in the ventricular fiber, in contrast to the marked decrease in the Purkinje fiber and in the nerve fiber. The results in the present experiment agreed well with that of JOHONSON ${ }^{6}$.

Change in electrotonic potential during the repolarization phase was studied by intermittent application of rectangular anodal pulses of $30 \mathrm{msec}$
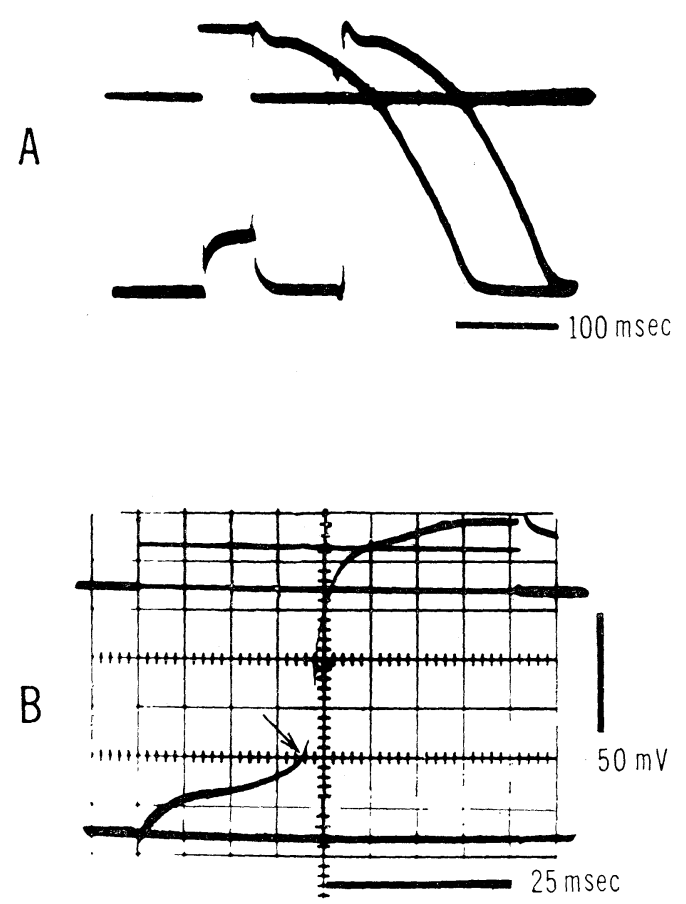

FIG. 5. Critical membrane potential in ventricular fiber. A : Depolarization is induced in the diastolic phase between two driven action potentials. Both of critical depolarizations which are effective and not effective in producing fullsized action potential are shown superimposed. B: Critical depolarization (arrow) at high sweep speed. Polarizing current monitored above both in $\mathrm{A}$ and $\mathrm{B}$.

* In such experiment, the strength of the anodal current was kept rather small, otherwise the abolition of action potential which will be described later was likely to occur. 
A

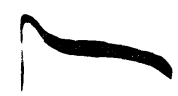

B

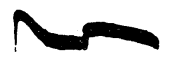

C

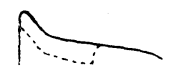

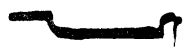
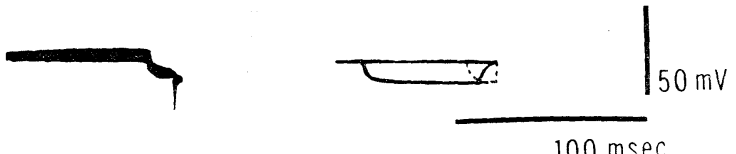

$100 \mathrm{msec}$

FIG. 6. Change in membrane resistance at the crest of action potential of ventricular fiber. A: Electrotonic potential $(10 \mathrm{mV})$ in resting phase of action potential. Overshoot is $+29 \mathrm{mV}$. B: Anodal current is applied $18 \mathrm{msec}$ before the upstroke of action potential until the initial portion of plateau. Overshoot is $20 \mathrm{mV}$. $\mathrm{C}$ : Superimposed tracing of $\mathrm{A}$ and $\mathrm{B}$.
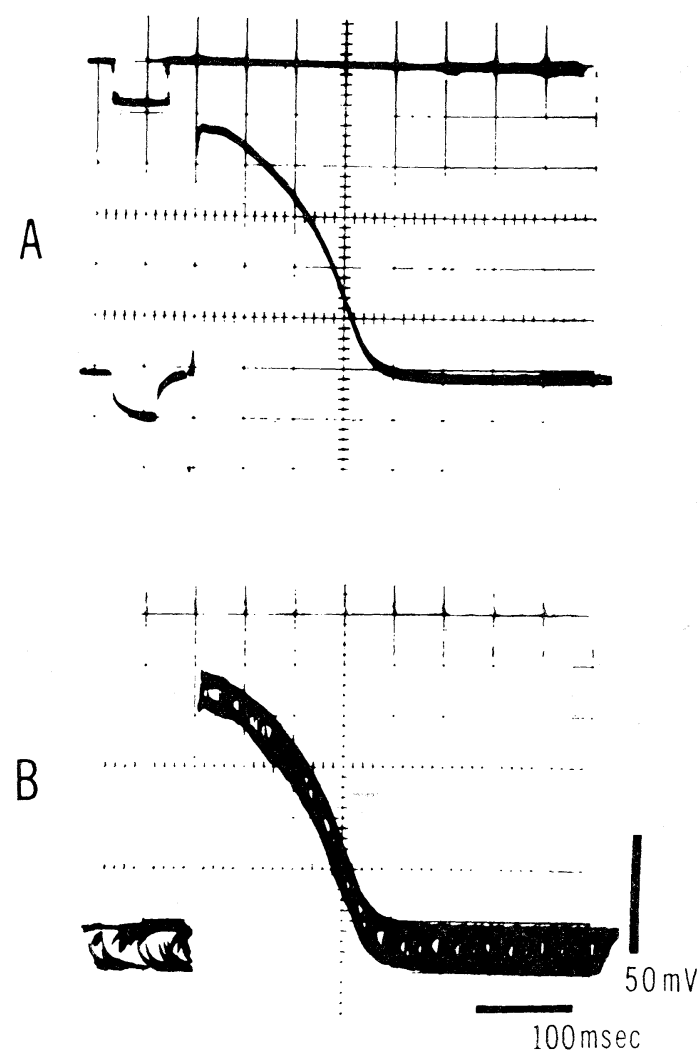

FIG. 7. Change in membrane resistance during repolarization phase in ventricular fiber. A : Electrotonic potential at resting phase of action potential. Above, monitor of current, $30 \mathrm{msec}$ in duration. B: Anodal polarization same as in A, applied repetitively at 10 c. p.s. Records of several sweeps superimposed. 
duration at 10 c.p.s.* An example of such record was shown in Fig. 7-B. The vertical width of the curve consisting of superimposed traces of several sweeps is proportional to the square root of membrane resistance. The record illustrates that the membrane resistance of the ventricular fiber throughout the repolarization phase remained almost the same as in the resting state. According to WEIDMANN, the membrane resistance in the Purkinje fiber increases to three times so high at the late phase of plateau of the resting value ${ }^{15)}$.

Abolition of action potential So-called abolition of action potential was reported to be elicitable by application of anodal current in the phase of plateau both in the Purkinje fiber ${ }^{15)}$ and the ventricular fiber $^{3)}$. On the other hand, JoHNSON and TILLE stated that the abolition could never be brought about in the ventricular fiber ${ }^{7 j}$. The present authors could not produce abolition except with extreme difficulty in the subendcardial terminal Purkinje fibers so far as the anodal current was passed through an intracellular electrode. Without doubt, it was impossible in the ventricular fiber with the same procedure.
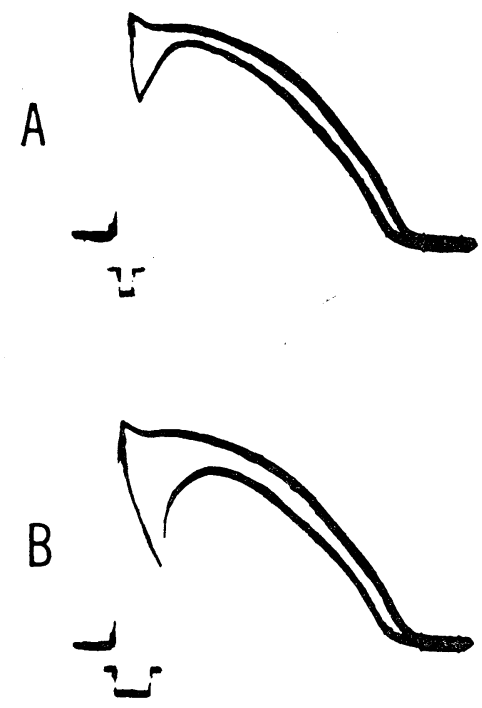

FIG. 8. Effect of brief anodal current (as shown below) at the early period of repolarization in the ventricular fiber. Incomplete abolition. Duration of polarizing current in A: $10 \mathrm{msec}, \mathrm{B}: 30 \mathrm{msec}, \mathrm{C}: 40 \mathrm{msec}$.

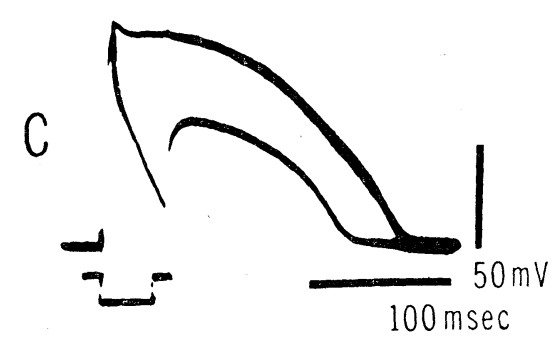




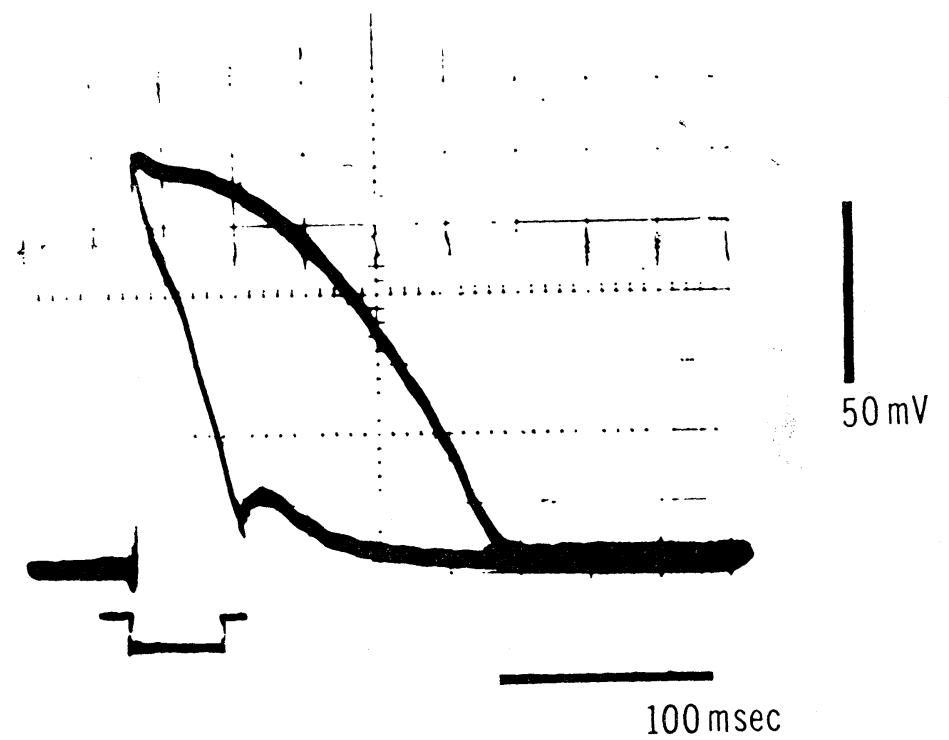

FIG. 9. "Abolition" of action potential in the ventricular fiber by anodal current of $50 \mathrm{msec}$ duration.

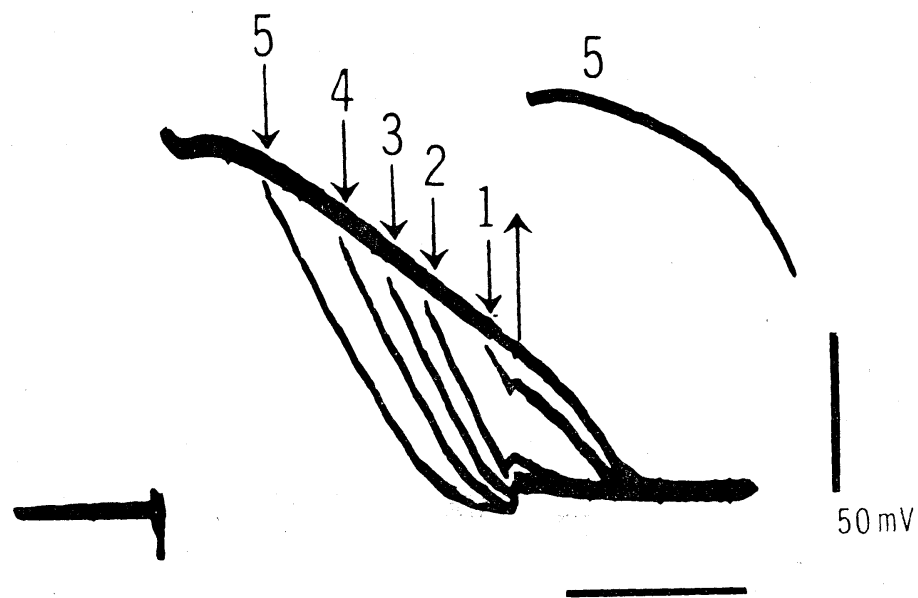

$100 \mathrm{msec}$

FIG. 10. Effects of anodal currents of various durations. Upward arrow : Fixed end point of anodal current pulses. Downward arrow : Starting points of currents of various durations (1,2, 3, 4, 5). Current 2 through 4 produced abolitions, current 5 was followed by post-anodal excitation. 
However, when the anodal current was applied by means of the separation method described above, it could produce abolition even in the ventricular fiber. In FIG. 8, anodal current of $10 \mathrm{msec}$ duration shortened the repolarization phase, even when the polarization was applied at the earliest phase of plateau immediately after the spike. In the case of FIG. 9, anodal current of $50 \mathrm{msec}$ duration produced a distinct abolition of action potential.

During the repolarization phase, the later the onset of anodal current and longer the duration of current, the more surely it produced abolition ${ }^{11}$. FIG. 10 illustrates such a fact. Here, the duration of anodal pulse was varied with its time of termination being fixed. While current 1 , which was started at the moment as indicated by arrow 1 and was shortest in duration, reduced slightly the duration of action potential, currents 2 through 4 , having more and more longer durations, abolished the action potential, and current 5 , the longest in duration, was followed by the post-anodal break excitation. This last mentioned response was reported already by BRooks et al. ${ }^{1)}$, WEIDMANN ${ }^{15}$ ) and Matsuda and Hoshi ${ }^{11}$.

\section{DISCUSSION}

The ventricular muscle fiber differs from the Purkinje fiber, not only in its smallness in diameter, but also in its fiber architectonics, i. e., its higher grade of fiber ramification and fiber-to-fiber anastomosis. Unlike in the Purkinje fibers, it is hardly possible to find a portion of the single fiber in the ventricular muscle without a branching at least for some length, which is suitable for electrophysiological study of its membrane properties. In fact, in the ventricular muscle, under usual circumstance, no measurable electrotonic potential could be recorded from the vicinity of the point source of polarizing current which was passed through an intracellular microelectrode. Electrotonic potentials could be observed in some cases, but its sizes were so small and its spatial decay was so marked that it is doubtful whether such findings could safely be utilized for estimating the membrane characteristics. The present authors' opinion is that such results are due to the fiber architecture in the ventricular muscle rather than the specific electrical property of the membrane. WOODBURY and CRILL ${ }^{16)}$ who had the similar results on the canine atrial muscle, attempted to explain it theoretically on an assumption that the atrium is a two dimensional fiber network. The ventricular muscle, however, should be regarded as a three dimensional network, and thus the spatial decay of electrotonic potential must be far pronounced. At any rate, the usual method of intracellular application of polarizing current can not effectively be employed for measuring precisely the spatial distribution of electrotonic potential. Were it be recorded, it needs to know the exact pattern of the network architecture in order to deduce the desired electrical constants from 
these experimental data.

For the purpose of circumventing these difficulties, the present authors attempted the new method for polarizing the ventricular muscle fibers. It might be permissible to assume that the ventricular fibers run parallel, and branch as well as anastomose with each other, both very densely but in the similar grade statistically. Thus they form a bundle as a whole. Suppose the polarizing current be applied simultaneously to all the fibers of such a bundle at its one end, the spatial distribution of the electrotonic potential would be almost equal to that in a single fiber having the same length and the same membrane characteristics. This conception was confirmed to be correct in the observations on some simulators for fiber networks constructed by the authors (unpublished results). The absolute value of the effective resistance of the ventricular fiber, of course, could not be determined by this method because the strength of current passed per fiber was unknown. But the course of the spatial decay of potential was measurable.

The present experiment demonstrated that the trend of the spatial decay of electrotonic potential could be made fit to the expected exponential law, from which the space constant $\lambda$ of the fiber was calculated to be $1.3 \mathrm{~mm}$ on the average. Though this value appeared to be slightly smaller than that in the Purkinje fiber which was reported to be $1.9 \mathrm{~mm}^{16)}$, both are of the similar order of magnitude. As the space constant will be proportional to the square root of the fiber diameter, it seems natural that the space constant of canine ventricular fiber which has the mean diameter of $16 \mu$ (MATSUDA, unpublished data) is smaller than that of the Purkinje fiber of sheep which has the mean diameter of $70 \mu$. The calculation $1.9 \times \sqrt{ } 16 / 70$ gives the expected value of $0.91 \mathrm{~mm}$ for the space constant of the ventricular fiber, which does not differ much from $1.3 \mathrm{~mm}$.

Whereas the liminal current for excitation was reported to be higher in the ventricular fiber than in the Purkinje fiber ${ }^{8)}$, no concrete experimental date have been presented so far concerning the critical membrane potential of the ventricular fiber. MATSUDA et al. did not succeed in eliciting the propagated action potential in the ventricular fiber by means of intracellular stimulation with a single microelectrode even with the maximum possible intensity and duration of current. If cathodal current, however, was applied simultaneously to many parallel fibers, as in the present experiment, not only the reasonable electrotonic potential but also the conductive action potential could easily be induced. The critical membrane potential which was estimated as the level of the point of inflexion in the foot of spike at the threshold stimulation (FIG. 5) was $-65 \mathrm{mV}$ on the average, the figure very close to that in the Purkinje fiber ${ }^{9)}$. Thus there seems to be no essential difference in critical membrane potential between both types of fibers.

The membrane resistance at the peak of action potential was found to be 
only $10 \%$ less than that in the resting phase in the ventricular fiber. This result agreed well with that of JoHnSON et al. ${ }^{6}$. The significant difference in the rate of decrease in resistance at the maximum of overshoot between the Purkinje fiber and the ventricular fiber is apparent, but difficult to interpret. Either the resistance might have dropped to its minimum before the peak of spike (though it could not be demonstrated experimentally so far) owing to an earlier onset of inactivation of sodium carrier, or the maximum reduction of resistance in the depolarization phase of action potential might really be smaller because the sum of the increased membrane conductance of sodium and the decreaseed conductance of potassium is lower in the ventricular fiber that in the Purkinje fiber, whereas the conductance ratio of sodium and potassium is about the same in both types of fibers.

WeIDmann could abolish the action potential of the Purkinje fiber of sheep by anodal intracellular polarization ${ }^{15)}$. He could bring about it even at the very early stage of plateau. In the canine ventricular fiber, however, such was evidently unsuccessful with a single microelectrode. The authors verified that the situation was the same also in the ventricular fiber of the sheep. According to CRANEFIELD and HofFMAN, abolition took place in the canine ventricular fiber, though not so easily, if the anodal current was applied extracellularly through an electrode having a large diameter ${ }^{3)}$. MATSUDA and HosHi confirmed the results by CRANEFIELD et al., and noticed that the later the current was given in the plateau phase, the more effective it was in producing the abolition. But in the earliest stage of repolarization they failed to produce it however strong a current might be employed. In the present experiment, while an anodal current of $10 \mathrm{msec}$ duration passed just after the peak of spike, accelerated distinctly the termination of the expected repolarization-incomplete abolition, the current, the same in strength but longer in duration $(50 \mathrm{msec})$ could completely abolish the action potential. In view of these facts, the effect of anodal current on the membrane in the phase of repolarization is not essentially different in the ventricular fiber from that in the Purkinje fiber. The difference in behavior between both types of fibers in responding to a single minute focus of intracellular polarization would be more apparent that real, i. e., its reason being to be sought in the difference in their fiber architecture than in the difference in the nature of their membranes. With respect to regenerative depolarization or repolarization, very localized polarization of membrane in the ventricular fiber might be suppressed by a neutralizing or antagonizing effect of many anastomosed adjacent fibers.

Or it might due to the difference in the ultrastructure of the intercalated disc as HAMA pointed out recently ${ }^{4}$. According to him, the portions of the fused membrane in an intercalated disc which are probably the sites for electrotonic spread of current are found to more numerous in the Purkinje fiber than in the ventricular fiber. 
By the way, the factor of length (or area) for excitation proposed by YAMAGIWA ${ }^{19)}$ may deserve consideration. His theory states that the area of the membrane which initially depolarized above the critical membrane potential should be greater than a liminal size (factor of length) in order to induce the propagated excitation. In the three dimensional ventricular muscle, the area which has been superaliminally depolarized by the stimulating current from a single microelectrode might be too small to evoke the propagated action potential even with the maximum possible strength of current, because of too great leakage of current through extensive branching and interconnection of fibers.

\section{SUMMARY}

A certain electrophysiological features of the ventricular fiber have been investigated in the papillary muscle of the dog by the use of a special method for polarizing, extracellularly and simultaneously, a large number of its fibers.

1) The electrotonic potential and its spatial decay was found to conform with the cable law as if it were in a single fiber. The space and time constants of the ventricular fiber could be estimated from the slopes of lines denoting interrelationships between the amplitude and the half time of rise of electrotonic potential to distance respectively. The calculated space constant was $1.3 \mathrm{~mm}$, and the time constant was $2.0 \mathrm{msec}$.

2) The critical membrane potential was $-65 \mathrm{mV}$.

3) The membrane resistance was found to decrease slightly at the peak of spike (to not less than $90 \%$ of resting resistance), and to remain about the same during the entire phase of plateau in the resting phase.

4) The action potential in the ventricular fiber could be abolished by anodal current even in the early stage of repolarization. The effectiveness of anodal polarization varied depending on the duration of current pulse and the timing of application: the longer the duration and later its application, the higher was the efficiency for producing the abolition.

5) In general, there seems to be no essential difference in nature of membrane between the ventricular and Purkinje fibers. The observed differences in electrophysiological behaviors between the two appear to be dependent rather on the difference in their fiber architectonic.

The expense needed for this study was defrayed, in part, by the grant of Ministry of Education awarded to one of the authors (M).

\section{REFERENCES}

1) Brooks, Ch. McC., Cranefield, P. F., Hoffman, B. F. and Siebens, A. A. Anodal effects during the refractory period of cardiac muscle. J. Cell. Comp. Physiol., 48: 237, 1956. 
2) Coraboeuf, E., Zacout, F., Gargoull, Y.M. and Lapland, J. Mésure de la résistance membranaire du myocarde ventricularire de memmiférés au cours de l'activité. Compt. Rend. Acard. Sc., 246 : 2934, 1958.

3) Cranefield, P. F. And Hoffman, B. F. Propagated repolarization in heart muscle. J. Gen. Physiol., $41:$ 633, 1958.

4) HAmA, K. Personal communication.

5) Hodgkin, A. L. And Rushton, W.A.H. The electrical constants of a crustacean nerve fiber. Proc. Roy. Soc., B133: 444, 1946.

6) Johnson, E.A., Robertson, P.A. And Tille, J. Purkinje and ventricular membrane resistance during the rising phase of the action potential. Nature, 182: 1162, 1958.

7) Johnson, E. A. And Tille, J. Changes in polarization resistance during the repolarization phase of the rabbit ventricular action potential. Aust. J. Exp. Biol., $38: 509,1960$.

8) Johnson, E. A. And Tille, J. Investigations of the electrical properties of cardiac muscle fibers with the aid of intracellular double-barrelled electrode. J. Gen. Physiol., 44 : 443, 1961.

9) Matsuda, K. Some electrical properties of terminal Purkinje fibers of the heart. in "Electrical activity of single cell" edited by Katsuki, Y., p. 283-294, Igakushoin, Tokyo, 1960.

10) Matsuda, K. Cardiac " membrane potential” as the spatial summation of multiple cells. Jap. Heart Journal, 4 : 505, 1963.

11) Matsuda, K. And Hoshi, T. "Abolition" of action potential in heart muscle of the dog. J. Physiol. Soc. of Jap., $23: 466,1961$. (in Japanese)

12) Matsuda, K., Hoshi, T. and Kameyama, S. Cellular potentials of normal mammalian ventricle. Seitai no Kagaku, $7: 379$, 1956. (in Japanese)

13) Matsuda, K., Kamiyama, A., Hoshiyama, M. and Hoshi, T. Impulse conduction between terminal Purkinje fiber and ventricular fiber in canine ventricle. J. Physiol. Soc. of Jap., $26: 63,1964$. (in Japanese)

14) Trautwein, W. Electrophysiologie der Herzmuskelfaser. Ergebniss der Physiol., $51: 131,1961$.

15) WeIDMANN, S. Effect of current flow on the membrane potential of cardiac muscle. J. Physiol., $115: 227,1951$.

16) Weidmann, S. The electrical constants of Purkinje fiber. J. Physiol., 118: 348, 1952.

17) WeIDMAnN, S. The effect of the cardiac membrane potential on the rapid availability of the sodium carrying system. J. Physiol., 127: 213, 1955.

18) Woodbury, J.W. And CRill, W. E. On the problem of impulse conduction in the atrium. in "Nervous inhibition" edited by Florey, E., p. 124-135, Pergamon Press, Oxford, 1961.

19) YAMAGIWA, K. Length-to-length transmission as the basis of conduction and some related considerations about the laws of excitation. Jap. J. Physiol. 5: 131, 1955. 